\title{
Biochemical alteration of hepatic functions by histamine H3-receptor agonist and antagonist in immunized rabbits
}

\author{
Tripathi $\mathrm{T}^{1,2}$, Khan $\mathrm{AA}^{3}$, Shahid $\mathrm{M}^{4}$, Dwivedi $\mathrm{V}^{5}$, Sarkar $\mathrm{S}^{6}$, Siddiqui $\mathrm{M}^{1}$, Khan $\mathrm{HM}^{4}$, \\ Mahdi $\mathrm{AA}^{7}$, Khan $\mathrm{RA}^{8}$
}

Department of Biochemistry, Faculty of Medicine, Jawaharlal Nehru Medical College \& Hospital, Aligarh Muslim University, Aligarh, India. trivendra.tripathi@unthsc.edu

\begin{abstract}
Aim: The aim of our study was to investigate the functional roles of H3R agonist and antagonist in the development of hepatic functions impairment in immunized rabbits.

Methods: The study comprised of six groups containing 18 rabbits in each. Group-I (negative control) and groupII (positive control) received sterile distilled water intramuscularly while Group III-VI received histamine (100 $\mu \mathrm{gkg}^{-1}$, s.c.), R-[-]-a-methylhistamine (H3R-agonist, $10 \mu \mathrm{gkg}^{-1}$, s.c.), iodophenpropit (H3R-antagonist, $1 \mu \mathrm{gkg}^{-1}$, i.m.), and the combination of iodophenpropit $\left(1 \mu \mathrm{gkg}^{-1}\right.$, i.m.) plus histamine $\left(100 \mu^{2} \mathrm{gkg}^{-1}\right.$, s.c. $)$, respectively, b.i.d. ( 12 hours [ 8 am and $8 \mathrm{pm}]$ ) for 10 days. Groups II-VI were immunized on day 3 with intravenous injection of sheep red blood cells $\left(1 \times 10^{9}\right.$ cells $\left./ \mathrm{ml}\right)$.

Results: On each experimental day, the mean values of serum enzymes and bilirubin in group-I and group-II showed no changes while in groups III, IV, V, and VI, these enzymes and bilirubin levels showed significant changes $(p<0.05)$, when compared with their values within the group. Profile of ALT and AST production revealed that ALT and AST levels moderately were changed due to degeneration of the liver.

Conclusion: Our results suggest that R-[-]- $\alpha$-methylhistamine showed moderate, and histamine and iodophenpropit showed mild degeneration of liver functions; while iodophenpropit plus histamine showed hepatic functions similar to control group. This study suggests that H3R antagonist in combination with histamine may be a non-toxic therapeutic target for histamine research (Fig. 7, Ref. 28). Text in PDF www.elis.sk.

Key words: histamine, histamine $\mathrm{H} 3$ receptor, agonist, antagonist, R-[-]- $\alpha$-methylhistamine, iodophenpropit, liver function test.
\end{abstract}

'Department of Biochemistry, Faculty of Medicine, Jawaharlal Nehru
Medical College \& Hospital, Aligarh Muslim University, Aligarh, In-
dia, ${ }^{2}$ Department of Cell Biology and Anatomy, University of North
Texas Health Science Center, Fort Worth, USA, ${ }^{3}$ Department of
Anatomy, Faculty of Medicine, Jawaharlal Nehru Medical College
and Hospital, Aligarh Muslim University, Aligarh, India, ${ }^{4}$ Depart-
ment of Microbiology, Faculty of Medicine, Jawaharlal Nehru Medi-
cal College \& Hospital, Aligarh Muslim University, Aligarh, India,
${ }^{5}$ Food Animal Health Research Program, Ohio Agricultural Research
and Development Center, The Ohio State University, 1680 Madison
Avenue, Wooster, USA ${ }^{6}$ Department of Biostatistics, University of
North Texas Health Science Center, Fort Worth, USA, ${ }^{7}$ Department
of Biochemistry, Chhatrapati Shahuji Maharaj Medical University,
Lucknow, India, and ${ }^{8}$ Department of Pharmacology, Faculty of Medi-
cine, Jawaharlal Nehru Medical College \& Hospital, Aligarh Muslim
University, Aligarh, India

Address for correspondence: T. Tripathi, PhD, Department of Cell Biology and Anatomy, University of North Texas Health Science, Center, 3500 Camp Bowie Blvd., Fort Worth, TX-76107, USA.

Phone: +1.817 .9088371$

Acknowledgements: T. Tripathi acknowledges University Grants Commission, New Delhi, India for providing UGC Fellowship [UGC letter DON F. 19-33/2006 (CU)]. The authors wish to thank Tocris Bioscience, Tocris Cookson Ltd. (United Kingdom) for kindly donating highly potent and specific histamine receptors-agonists and antagonists. We thank Prof. Abida Malik, Incharge, Central Animal House, J.N.M.C., Aligarh Muslim University, Aligarh for providing animal facilities.
Health-care providers know that sneezing, wheezing, and itching are common health problems that most often occur by due to a small molecule, namely, histamine (1). Histamine mediates several functions by four distinct cell membrane receptors H1R, $\mathrm{H} 2 \mathrm{R}, \mathrm{H} 3 \mathrm{R}$, and H4R (2). It shows agonist property and has different $\mathrm{p} K \mathrm{i}$ values of its receptors, $4.2 \pm 0.1$ for H1R, $4.3 \pm 0.1$ for $\mathrm{H} 2 \mathrm{R}, 7.8 \pm 0.1$ for $\mathrm{H} 3 \mathrm{R}$, and $8.1 \pm 0.1$ for H4R. Histamine receptors (HRs) belong to the G-protein-coupled receptor (GPCR) family as Gq/11 for H1R, Gas for H2R, Gi/o for H3R, and H4R (3). H1R is associated with wakefulness and inflammatory response; H2R is responsible for gastric acid secretion; H3R controls the release of histamine and other neuromediators in the CNS. The role of H4R is currently unclear although preclinical evidence suggests its potential role in inflammation and pain processes (4). The antagonists of H1R and H2R have been introduced into market for a long time (2). Moreover, histamine H3R and H4R antagonists are currently in preclinical development and in early stages of clinical trials for a range of cognitive, psychotic, sleep and inflammatory disorders, obesity and cancers (5). It has been assumed that within the next few years H3R and H4R antagonists will be freely available in the market. Moreover, H3R has been found to have a role in allergic rhinitis. Its expression has been demonstrated to be present around the submucosal glands in human nasal tis- 
$675-681$

sue. Thioperamide has been shown to cause degranulation of mucus granules in the submucosal glands. It is assumed that, by inhibiting H3R, acetylcholine is released in the nose, leading to degranulation of the mucosal glands and mucus secretion. These results suggest that there may be a role for $\mathrm{H} 3 \mathrm{R}$ agonists in the treatment of rhinitis $(1,6)$. The effects of H3R agonists and antagonists have been demonstrated in the treatment of pain, headache, and even asthma (1). Although the physiological and pathological relevance of histamine receptors (HR) and their distribution in various tissues are being documented (2), the exact role of various HR agonists and antagonists on liver functions are still unknown. Our previous studies have shown immunomodulatory and hepatotoxic roles of HR agonists and antagonists (7-11). While, in vivo effects of histamine, pheniramine (H1R-antagonist), histamine trifluoro-methyl toluidide (H1R-agonist), ranitidine (H2Rantagonist) and amthamine (H2R-agonist) in immunized animals demonstrated impaired hepatic functions (12-14). These findings, along with our previous studies of HR agonists and antagonists (7-10) demonstrate that though HR agonists and antagonists are mildly hepatotoxic yet these might be useful for immune targeted diseases. Therefore, we have designed hepatic studies to explore the degree of hepatotoxicity of each histamine receptor's agonist and antagonist that were used in our previous immunotoxic studies. Preliminary studies of H1R and H2R -agonist and antagonist have shown different patterns of hepatotoxicity (12-14); in addition to determine the toxic effects of histamine receptor's ligands, the present study was planned to investigate the functional role of histamine H3R agonist (R-[-]- $\alpha$-methylhistamine) and antagonist (Iodophenpropit) in the development of liver function impairment in immunized rabbits.

\section{Materials and methods}

\section{Animals}

To evaluate the hepatotoxicity of H3R-agonist and antagonist, New Zealand adult healthy albino rabbits of either sex weighing $1.0-1.5 \mathrm{~kg}$ were randomized equally into experimental groups. They were housed in a well maintained animal facility at Central Animal House, J. N. Medical College, Aligarh Muslim University, Aligarh, in the Bio resources unit under $12 \mathrm{hrs} \mathrm{light/dark} \mathrm{cycle,}$ temperature $\left(22 \pm 2{ }^{\circ} \mathrm{C}\right)$ and were allowed free access to standard laboratory diet, including green vegetables, and tap water until experimentation. All the studies were carried out during the light cycle and were approved by the Institutional Animal Ethical Committee.

\section{Selection of specific dose of H3R-antagonist (Iodophenpropit)}

To determine the non-toxic specific dose of iodophenpropit for in vivo animal experiments, the dose-dependent study of iodophenpropit was designed. Group-A was vehicle-treated (sterile distilled water and served as control) and group B-D treated with iodophenpropit dihydrobromide at doses of 1,5 , and $10 \mu \mathrm{g} / \mathrm{kg}$, respectively, two times a day [ 12 hourly ( 8 am and $8 \mathrm{pm}$ ) through intramuscular (i.m.) route. Each group contained three non-immunized rabbits $(n=3)$. Dose-dependent activity was investigated by liver histopa- thology. Rabbits in group A and B were sacrificed by high dose ether anesthesia after 10 days of drug administration. However, all rabbits of group C and D were found dead at day 2 after drug administration. Livers of all animals were immediately perfused with $10 \%$ buffered formalin. $10 \mathrm{~mm} \times 5 \mathrm{~mm} \times 3 \mathrm{~mm}$ blocks of liver were processed for paraffin embedding. $10 \mu \mathrm{m}$ thick paraffin sections were stained with haematoxylin and eosin, and were examined under light microscope (Olympus-BX40-Japan). Observations were recorded in sample photomicrographs taken at $200 \times$.

\section{Biochemical study of hepatic functions}

\section{Antigen, immunization and drug dosage regimen}

Sheep red blood cells (SRBC) were isolated and characterized as reported previously (3-8). Group I (negative control) and Group II (positive control) received vehicle (sterile distilled water, $1 \mathrm{ml} /$ $\mathrm{kg}$, b.i.d.). Groups III, IV, V, and VI were treated with histamine, H3R agonist, H3R antagonist, and H3R antagonist + histamine (Histamine was given 30 minutes before of H3R antagonist administration), respectively. All rabbits in the groups (II-VI) were immunized on day 3 intravenously (i.v.) with $1 \mathrm{ml}$ of $5 \%$ ( $1 \mathrm{x}$ $10^{9}$ cells $/ \mathrm{ml}$ ) SRBC in PBS. Each group (I-VI) contained eighteen rabbits $(\mathrm{n}=18)$. Histamine dihydrochloride, $100 \mu \mathrm{gkg}^{-1}$ and R-[-]$\alpha$-methylhistamine dihydrobromide, $10 \mu \mathrm{gkg}^{-1}$ were administered through subcutaneous (s.c.) route, and iodophenpropit dihydrobromide, $1 \mu \mathrm{gkg}^{-1}$ was administered through intramuscular (i.m.) route twice a day [ 12 hourly ( 8 am and $8 \mathrm{pm}$ )] for 10 days (starting from 3 days prior to immunization until 7 days after immunization). All doses referred to the weight of the salts used. Histamine dihydrochloride was purchased from Himedia Laboratories Pvt. Limited, India. H3R agonist (R-[-]- $\alpha$-methylhistamine dihydrobromide) and H3R antagonist (Iodophenpropit dihydrobromide) were kindly donated by Tocris Bioscience, Tocris Cookson Ltd., United Kingdom.

\section{Biochemical analyses}

To determine the biochemical levels for liver function, blood samples were collected from rabbits through the marginal ear veins prior to immunization (day 0), as well as on days $7,14,21$, 28 , and 58 after immunization. Blood samples were kept at room temperature for $2 \mathrm{hrs}$ and then at $4{ }^{\circ} \mathrm{C}$ ovennight. Blood samples were centrifuged for $10 \mathrm{~min}$ at $580 \mathrm{xg}$, and serum was separated and heated at $56^{\circ} \mathrm{C}$ for $30 \mathrm{~min}$ to inactivate the complement proteins and stored in aliquots containing sodium azide as a preservative at $-20^{\circ} \mathrm{C}$. Serum levels of liver enzymes, including aspartate aminotransferase (AST), alanine aminotransferase (ALT), alkaline phosphatase (ALP), and bilirubin [total bilirubin (TB), direct bilirubin (DB), and indirect bilirubin (ID)] were determined using an automatic analyzer (Transasia XL 300, Germany).

\section{Statistical analyses}

Data were summarized as Mean $\pm \mathrm{SD}$. Groups were compared by using repeated measures (subjects within groups) two way analysis of variance (ANOVA) followed by Newman-Keuls post hoc test. $\mathrm{p}<0.05$ was considered to be statistically significant. 

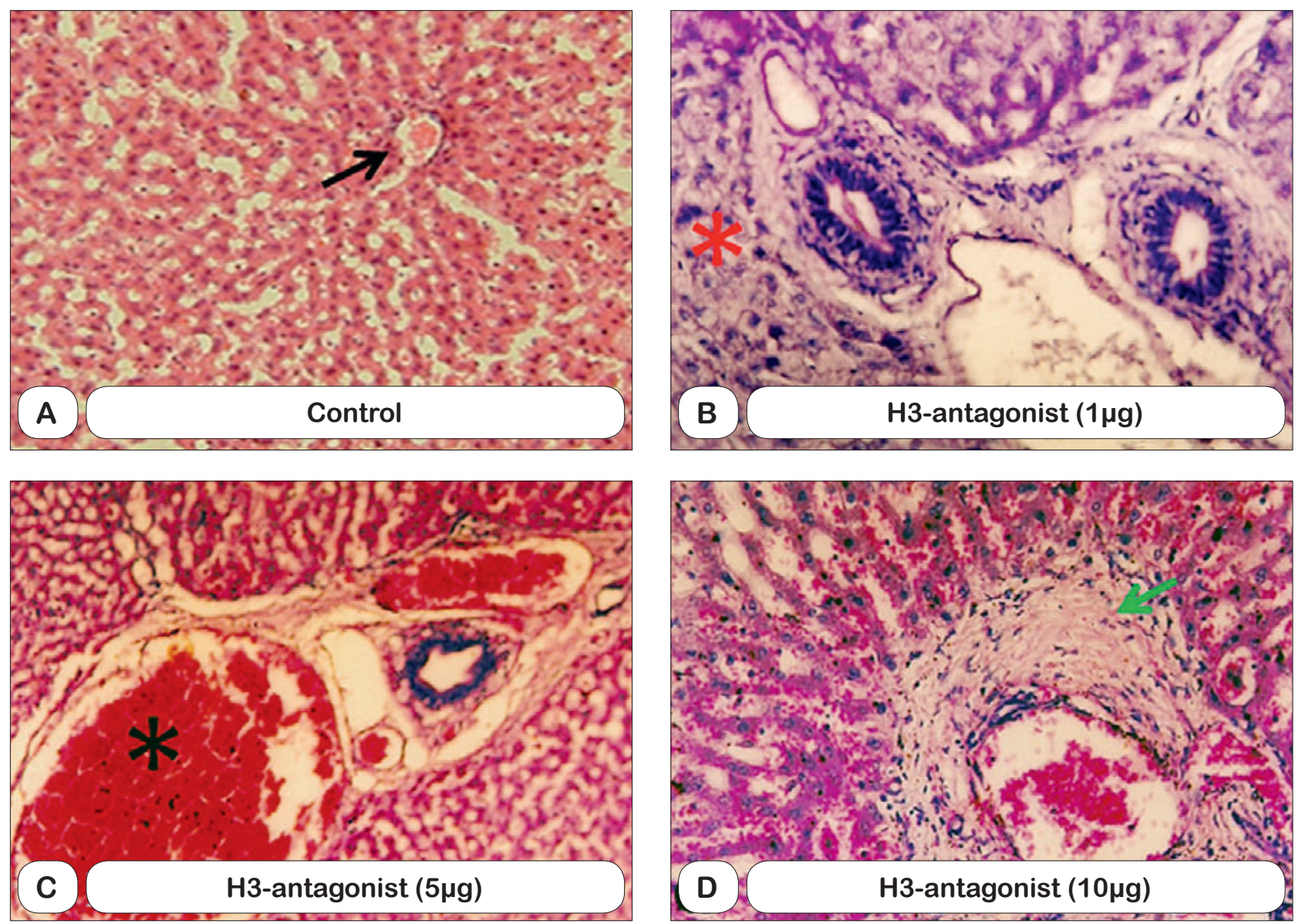

Fig. 1. Photomicrographs from control group (a) showing central vein (arrow), normal radial arrangement of hepatic cords and intervening sinusoids; H3R-antagonist, $(1 \mu \mathrm{g} / \mathrm{kg})(\mathrm{b})$ reveals distortion of normal cytoarchitecture of hepatocytes and focal degenerative changes $(*)$ and portal triads with periportal lymphocytic infiltration; H3R-antagonist $(5 \mu \mathrm{g} / \mathrm{kg})$ (c) shows congested veins and sinusoids with moderate distortion of hepatic cytoarchitecture; H3R-antagonist $(10 \mu \mathrm{g} / \mathrm{kg})(\mathrm{d})$ demonstrates congested veins and sinusoids with periportal fibrosis (arrow), and focal hepatic degeneration. Haematoxylin and eosin; $\mathbf{x 2 0 0}$.

\section{Results}

\section{Histopathological examination}

The microscopic findings showed that Groups B (Fig. 1b), C (Fig. 1c), and D (Fig. 1d) have congested and dilated sinusoids and degenerative changes in the hepatocytes as compared to Group A (Fig. 1a), this clearly suggest that the changes are related to treatment with iodophenpropit. The microscopic findings of Group B showed mild congestion and degeneration while that of Group $C$ and D showed a severe degree of hepatic necrosis, portal congestion and periportal fibrosis. It is evident that the findings in Groups $C$ and $D$ cause irreversible toxic damages to the liver leading to the death of the rabbits. The dosage regimen of our study required to administer H3R antagonist for a period of 10 days, thus as H3R antagonist given at dose of $5 \mu \mathrm{g} / \mathrm{kg}$ and $10 \mu \mathrm{g} / \mathrm{kg}$ leads to death of the rabbit due to toxic liver damage 10 days before the drug schedule, we chose 1 $\mu \mathrm{g} / \mathrm{kg}$ dose of iodophenpropit for immunotoxic study. Also, we observed that though 10 days and 30 days administration of H3R-agonist (R-(-)- $\alpha$-methylhistamine) with the dose of $10 \mu \mathrm{g} / \mathrm{kg}$ showed mild to moderate hepatotoxicity yet this dose was tolerated by rabbits and all treated rabbits were alive during long-term administration (10-11). Thus, we decided to use the $10 \mu \mathrm{g} / \mathrm{kg}$ dose of R-(-)- $\alpha-$ methylhistamine for in vivo biochemical and immunotoxic study.

\section{Biochemical examination}

R-[-]- $\alpha$-methylhistamine treatment moderately increased ALT and AST levels when compared to rest of the group on days 0,7 , $14,21,28$, and 58 after immunization. Iodophenpropit treatment increased ALT and AST levels on day 14, 21, 28, and 58 after immunization when compared to the groups of iodophenpropit plus histamine, histamine, positive control, and negative control; while, it showed decreased levels of ALT and AST as compared to R-[-]$\alpha$-methylhistamine group (Fig. 2 and 3). Profile of ALT and AST production revealed that the ALT and AST levels moderately increased in the groups: R-[-]- $\alpha$-methylhistamine > iodophenpropit $>$ iodophenpropit plus histamine $=$ histamine $=$ positive control $=$ negative control. Moreover, histamine treatment showed significantly increased level of ALP when compared to rest of the 


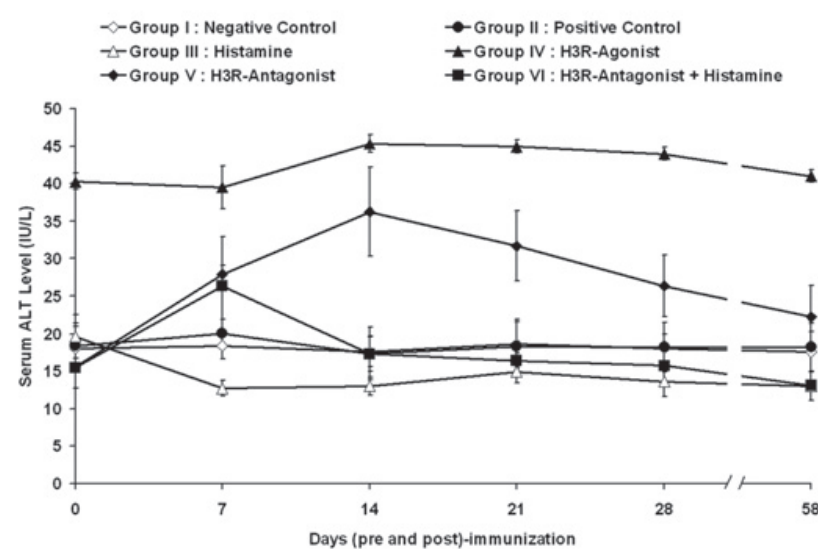

Fig. 2. Effects of histamine, H3R-agonist (R-[-]- $\alpha$-methylhistamine) and H3R-antagonist (Iodophenpropit) on serum ALT levels. The results demonstrate mean \pm S.D. of three experiments each with six rabbits. Two-way ANOVA followed by Newman-Keuls post hoc test revealed that the effects of treatments $(F=10935.595, D F=5.102 ; p<0.01)$ and days $(F=32.063, D F=5.510 ; p<0.01)$ on $S R B C$ were statistically significant. The interaction (treatments $x$ days) effects of $(F=56.478$, $D F=25.510 ; p<0.01)$ these on SRBC were also found to be significant.

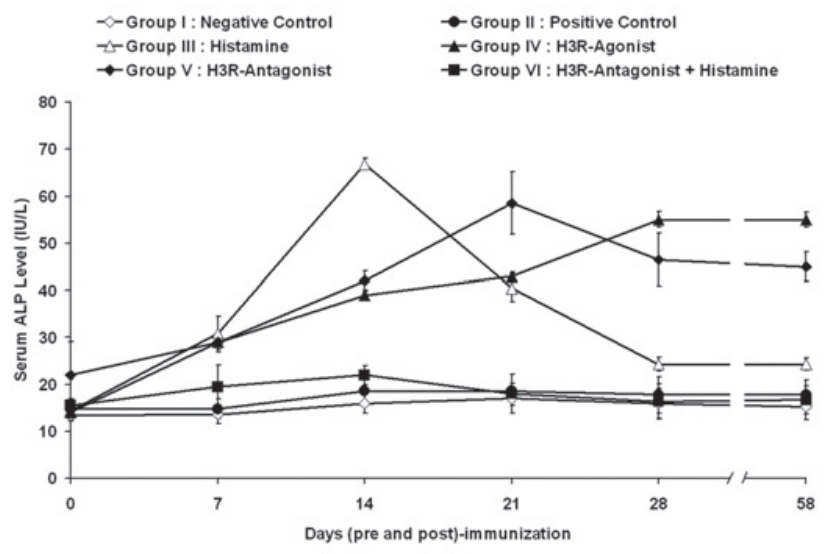

Fig. 4. Effects of histamine, H3R-agonist (R-[-]- $\alpha$-methylhistamine) and H3R-antagonist (Iodophenpropit) on serum alkaline phosphatase (ALP) levels. The results demonstrate mean \pm S.D. of three experiments each with six rabbits. Two-way ANOVA followed by Newman-Keuls post hoc test revealed that the effects of treatments $(F=35548.234$, $D F=5.102$; $\mathrm{p}<0.01)$ and days $(\mathrm{F}=\mathbf{5 4 5 . 7 8 3}, \mathrm{DF}=\mathbf{5 . 5 1 0} ; \mathbf{p}<\mathbf{0 . 0 1})$ on $\mathrm{SRBC}$ were statistically significant. The interaction (treatments $x$ days) effect of $(F=265.478$, $D F=25.510 ; p<0.01)$ these on SRBC were also found to be significant.

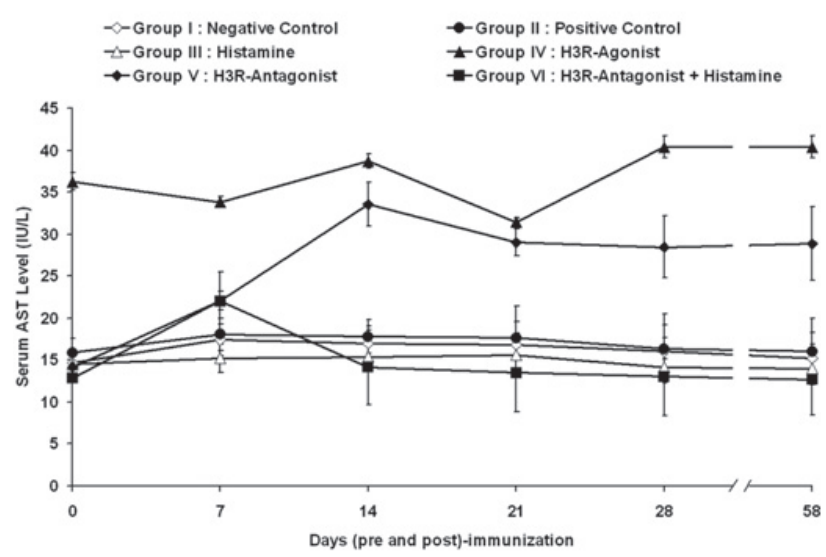

Fig. 3. Effects of histamine, H3R-agonist (R-[-]- $\alpha$-methylhistamine) and H3R-antagonist (Iodophenpropit) on serum AST levels. The results demonstrate mean \pm S.D. of three experiments each with six rabbits. Two-way ANOVA followed by Newman-Keuls post hoc test revealed that the effect of treatments $(\mathrm{F}=12671.698, \mathrm{DF}=5.102 ; \mathrm{p}<0.01)$ and days $(F=30.301, D F=5.510 ; p<0.01)$ on $S R B C$ were statistically significant. The interaction (treatments $x$ days) effects of $(F=24.980, D F=25.510$; $\mathbf{p}<0.01$ ) these on SRBC were also found to be significant.

groups on day 14 after immunization. However, it showed decreased level of ALP on days 21, 28, and 58 after immunization when compared to R-[-]- $\alpha$-methylhistamine and iodophenpropit. While, R-[-]- $\alpha$-methylhistamine and iodophenpropit revealed significantly increased ALP levels on day 7, 14, 21, 28, and 58 after immunization when compared to iodophenpropit plus histamine, positive control, and negative control (Fig. 4). In addition to the bilirubin, total bilirubin (TB), direct bilirubin (DB), and indirect bilirubin (IB) findings demonstrated that R-[-]- $\alpha$-methylhistamine and histamine showed impaired liver functions when compared

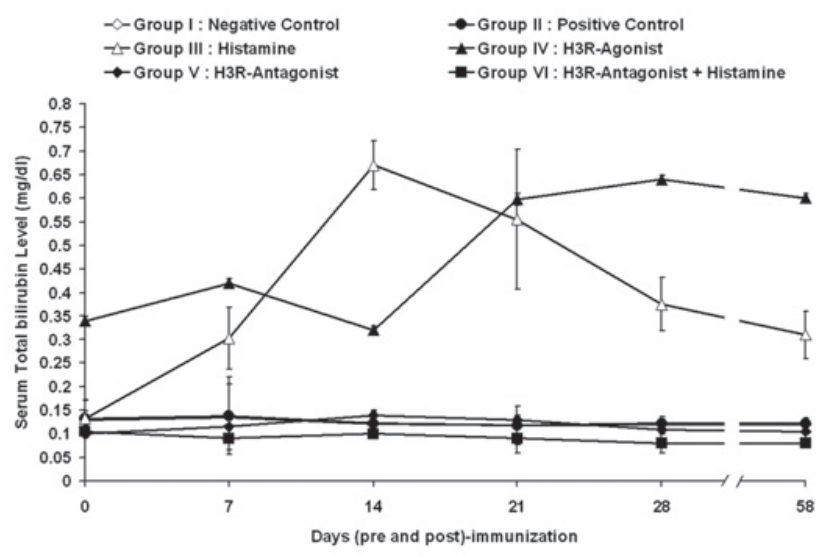

Fig. 5. Effects of histamine, H3R-agonist (R-[-]-a-methylhistamine), and H3R-antagonist (Iodophenpropit) on serum total bilirubin (TB) levels. The results demonstrate mean \pm S.D. of three experiments each with six rabbits. Two-way ANOVA followed by Newman-Keuls post hoc test revealed that the effects of treatments $(F=20284.218, D F=5.102 ; p<0.01)$ and days $(F=110.851, D F=5.510 ; p<0.01)$ on $S R B C$ were statistically significant. The interaction (treatments $x$ days) effects of $(F=114.671$, $D F=25.510 ; p<0.01$ ) these on SRBC were also found to be significant.

with iodophenpropit, positive control and negative control (Fig. 5-7). The production profile of TB, DB, and IB showed hepatic degeneration in the treatment groups of R-[-]- $\alpha$-methylhistamine $>$ histamine $>$ positive control $=$ negative control $=$ iodophenpropit $=$ iodophenpropit plus histamine.

\section{Discussion}

Immunopharmacological studies have demonstrated immunotoxic impact of drugs and chemicals using immunized animals 


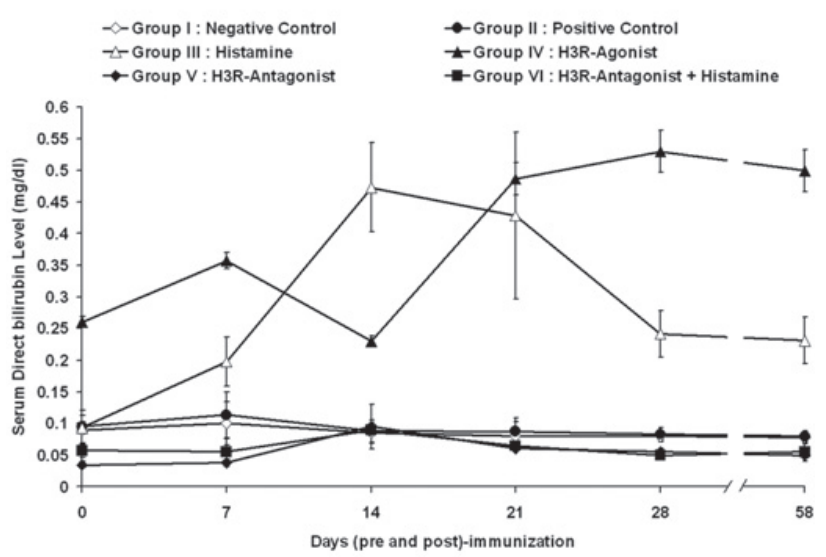

Fig. 6. Effects of histamine, H3R-agonist (R-[-]- $\alpha$-methylhistamine), and H3R-antagonist (Iodophenpropit) on serum direct bilirubin (DB) levels. The results demonstrate mean \pm S.D. of three experiments each with six rabbits. Two-way ANOVA followed by Newman-Keuls post hoc test revealed that the effects of treatments $(F=15751.856, D F=5.102 ; p<0.01)$ and days $(F=98.260, D F=5.510 ; p<0.01)$ on $S R B C$ were statistically significant. The interaction (treatments $x$ days) effects of $(F=121.788$, $D F=25.510 ; p<0.01)$ these on SRBC were also found to be significant.

(15-17). However, the impacts of immunotoxic studies on liver functions are still to be elucidated. Histamine is a natural mediator of allergic adverse effect. It participates in the regulation of arousal and circadian rhythmicity, indicating that induction of central histamine mechanisms may contribute to the development of neuropsychiatric symptoms, such as sleep disturbances and altered circadian rhythms in chronic hepatic encephalopathy (HE), and suggest that pharmacological manipulation of the histaminergic system could be beneficial in the treatment of HE in chronic liver failure (18). H3R plays an important role in HE. Previous study demonstrated that binding density of H3R sites decreased together with increased histamine metabolite concentrations in the brain of cirrhotic patients suggesting an inability of the autoregulatory $\mathrm{H} 3 \mathrm{R}$ to tightly control the signaling cascades regulating histamine release in HE (18). It has been shown that continuous exposure to high histamine concentrations may lead to decreased number of receptor sites consistent with a selective down-regulation of H3R. Thus, H3R stimulation inhibits both the synthesis and release of histamine (19-20). In an in vivo study, administration of (R)-(-)- $\alpha-$ methylhistamine (H3R-agonist) to bile duct-ligated (BDL) rats decreased cholangiocyte growth to a higher extent compared to histamine and suggested that endogenous histamine also interacts with other HRs, exerting an opposite, pro-proliferative effect. However, activation of H1R and H2R enhances normal cholangiocyte growth (21). In contrast when BDL rats were administered with the H3R-antagonist (Thioperamide), increase of bile duct mass in reaction to cholestasis was higher than BDL rats (21). In addition, (R)-(-)- $\alpha$-methylhistamine ameliorates lobular damage and decreases the serum levels of bilirubin and AST compared to BDL rats treated with $\mathrm{NaCl}$. Thus, this study indicates that the excessive proliferation of cholangiocytes in the course of cholestatic liver diseases could be counterbalanced by the selective activation of

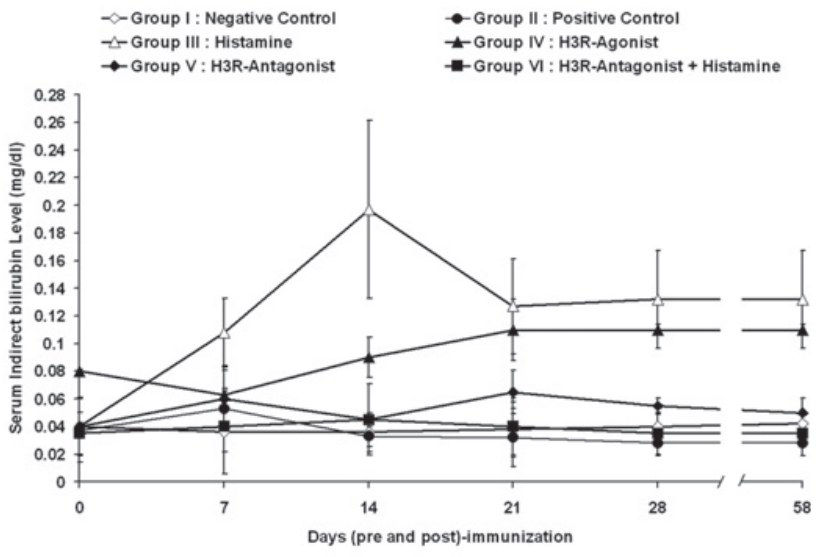

Fig. 7. Effects of histamine, H3R-agonist (R-[-]- $\alpha$-methylhistamine), and H3R-antagonist (Iodophenpropit) on serum indirect bilirubin (IB) levels. The results demonstrate mean \pm S.D. of three experiments each with six rabbits. Two-way ANOVA followed by Newman-Keuls post hoc test revealed that the effects of treatments $(F=1195.783, D F=5.102$; $p<0.01)$ and days $(F=1.8156, D F=5.510 ; p=0.1)$ on $S R B C$ were statistically significant. The interaction (treatments $x$ days) effects of $(F=4.070$, $D F=25.510 ; p<0.01$ ) these on SRBC were also found to be significant.

H3R, which mediates inhibition of intrahepatic ductal mass (21). In addition to control, the role of histamine synthesis and release, H3R modulates other neurotransmitters such as catecholamines and serotonin (22) and thus further contributes to the development of HE via mechanisms involving these amines. Keeping in mind that human disease processes mediated by histamine release may potentially be modified by the use of selective H3R agonists and antagonists, the hepatotoxic study of H3R agonist and antagonist in immunized condition has been determined to demarcate the effects of short-term exposure to H3R agonist and antagonist on liver functions. Abnormalities in liver function tests are increased levels of ALT, AST, ALP, and bilirubin (23). Mild to moderate elevations in serum ALT and AST levels may be observed in virtually all liver diseases. Therefore, ALT and AST levels beyond 15 times the upper limits of normal are usually considered significant degree of hepatic dysfunctions, and this degree is generally found in few conditions like hepatitis (drug-induced, acute viral, ischemic and autoimmune), common bile duct stones and muscle injury (24-26). It was proven in previous research that H3R agonist inhibits the release and synthesis of histamine. It could be possible that due to autoregulation, there is a feedback increase of catecholamine's serotonin and dopamine, as H3Rs are known to regulate serotonin and dopamine also. The increase in these catecholamines would be detrimental to the liver and in turn increase the levels of AST and ALT. When an H3R antagonist is used, it increases the levels of histamine in the cell which itself proves as toxic to the liver and increases AST and ALT (18-21). AST and ALT results of our study showed that when histamine alone was used the ALT and AST levels were the same as control suggesting that exogenous histamine itself is not toxic to the liver, as previously stated H3R agonist inhibits both the release and synthesis of histamine (18-21), this might autoregulate the activity 
of H1R, H2R, and H4R leading to an increase of AST and ALT levels, whereas H3R inhibition stimulates both the synthesis and the release of histamine in vivo, which indicates that continuous exposure to high histamine concentrations may lead to decrease in the number of receptor sites consistent with a selective down regulation of $\mathrm{H} 3 \mathrm{R}$ and an up-regulation of $\mathrm{H} 1 \mathrm{R}, \mathrm{H} 2 \mathrm{R}$, and H4R; this could be a cause of an increase in the AST and ALT levels (18-21). In addition to determine whether in vivo histamine synthesis is harmful to the liver we used histamine along with a H3R antagonist (An indirect H3R agonist study). In this case because all HR are already activated with exogenous histamine, when H3R antagonist was added the auto regulation of the other HR did not lead to the increase in ALT and AST. This demonstrates that the H3R antagonist with the combination of histamine does not affect the biochemical alteration of AST and ALT suggesting a suitable drug combination for in vivo clinical studies of H3R. Thus, these results may have clinical importance to study HR modulators in various immune mediating diseases warranting further studies.

Serum alkaline phosphatase (ALP) activity may derive from liver, bone, intestine, and placenta. Various hepatobiliary (such as drugs, bile duct obstruction, primary biliary cirrhosis, primary sclerosing cholangitis, liver metastasis, infiltrative liver disease, viral hepatitis, alcoholic hepatitis, and cirrhosis) and non-hepatic (bone disease, pregnancy, chronic renal failure, congestive heart failure, childhood growth, and malignancy) causes are related with raised ALP. However, low serum ALP in patients with biochemical evidence of liver dysfunction may be a clue to Wilson's disease. Also, low ALP may be encountered in hypothyroidism, zinc deficiency, pernicious anemia, and congenital hypophosphatemia (27). The ALP results of the current study showed that the ALP levels after treating with histamine, H3R agonist and antagonist increased as compared to the H3R antagonist plus histamine, positive control, and negative control. As the ALP levels are not a specific marker for liver injury, this increase could be either due to a liver problem induced by the drug or by other factors affecting the other body systems as mentioned above. Thus, the present findings also suggest that the combination of H3R antagonist and histamine does not show any alteration and it could be used to develop a suitable drug target for in vivo study of H3R.

Serum bilirubin is a mixture of $\alpha, \beta, \gamma$, and $\delta$ fragments which are unconjugated, singly conjugated, doubly conjugated and covalently bound to albumin, respectively (28). In most cases a total bilirubin (TB) assay suffices for LFT, but fractionation may be required for isolated increases in bilirubin and neonatal jaundice. Direct bilirubin (DB) refers to the conjugated bilirubin that reacts directly with the diazo- reagent, while indirect bilirubin (IB) is a derived value obtained from the difference of TB and DB. DB assays measure only $70-90 \%$ of the conjugated and $\delta$ bilirubin, and may underestimate the severity of jaundice (28). Fractionation of serum bilirubin is useful in separating the causes of jaundice. In prehepatic jaundice due to hemolysis, unconjugated bilirubin is increased with little or no increase in conjugated bilirubin. In hepatic and post-hepatic jaundice, there are increased conjugated and $\delta$ bilirubin levels (28). When the liver function tests are abnormal and the serum bilirubin levels are more than $17 \mu \mathrm{mol} / \mathrm{L}$ it suggest an underlying liver disease (23). Our results of direct bilirubin showed increased level of direct bilirubin (DB) as compared to indirect bilirubin (IB) in histamine and H3R agonist treated group which suggests a bilirubin alteration leading to hyperbilirubinemia. This study further suggests that the combination of H3R antagonist with histamine does not show any effect on bilirubin alteration as compared to positive control and negative control. Thus, such combinations may be suitable for drug targeting studies in immune related disorders. We also noticed that the liver enzymes and bilirubin levels were not affected by the treatment with sterile distilled water in SRBC-immunized (positive control) or unimmunized (negative control) groups. The results of these groups are the same as our previous findings (12-14) and thus, this study revealed that the $\mathrm{SRBC}$ is not hepatotoxic and is a suitable antigen for immunotoxic studies.

\section{Conclusion}

The biochemical examinations of liver enzymes and bilirubin revealed that short-term treatment of R-[-]- $\alpha$-methylhistamine showed moderate differential patterns of hepatic dysfunctions. Histamine and iodophenpropit showed mild disturbances of liver functions; while iodophenpropit plus histamine showed hepatic functions similar to control levels. This suggests that the combination of an antagonist with histamine may potentially be modified for the use in the treatment of human immune mediated diseases.

\section{References}

1. Jones BL, Kearns GL. Histamine: new thoughts about a familiar mediator. Clin Pharmacol Ther 2011; 89: 89-97.

2. Shahid M, Khardori N, Khan RA, Tripathi T (Eds). Biomedical aspects of histamine: Current perspectives. Springer Heidelberg, The Netherlands, 2010.

3. Huang JF, Thurmond RL. The new biology of histamine receptors. Curr Allergy Asthma Rep 2008; 8: 21-27.

4. Thurmond RL, Gelfand EW, Dunford PJ. The role of histamine H1 and $\mathrm{H} 4$ receptors in allergic inflammation: the search for new antihistamines. Nat Rev Drug Discov 2008; 7: 41-53.

5. Chazot PL. Advances in histamine pharmacology reveal new drug targets. British J Pharmacol 2009; 157: 1-3.

6. Suzuki S, Takeuchi K, Majima Y. Localization and function of histamine H3 receptor in the nasal mucosa. Clin Exp Allergy 2008; 38 : 1476-1482.

7. Tripathi T, Shahid M, Raza A et al. In vivo comparative immunotoxic study of histamine receptors (H1R, H2R, H3R and H4R)-agonist. Eastern J Med 2010; 15: 48-56.

8. Tripathi T, Shahid M, Khan HM et al. Modulation of in vivo immunoglobulin synthesis by endogenous histamine and by H1R- and H2Ragonists and antagonists. Pharmacol Reports 2010; 62: 917-925.

9. Tripathi T, Khan AA, Shahid M, et al. Immunological, biochemical and histopathological evaluation of histamine receptors (H1R, H2R, H3R and H4R)-antagonist in rabbit experimental model: a short term study. Exp Toxicol Pathol 2010; 64: 259-266. 
10. Tripathi T, Khan AA, Shahid M et al. Biochemical and histopathological evaluation of histamine receptors (H1R, H2R, H3R and H4R)agonist in rabbits. Exp Toxicol Pathol 2013; 65: 271-275.

11. Tripathi T, Khan AA, Shahid M et al. Hepatotoxicity Due to Histamine Trifluoro-Methyl Toluidide, Amthamine, R-(-)- $\alpha$-Methyl Histamine and Clobenpropit (H1R-H4R-Agonists, Respectively) in Rabbit Experimental Model. Am Med J 2010; 1: 1-7.

12. Tripathi T, Shahid M, Khan HM et al. Role of histamine $\mathrm{H} 2$ receptoragonist/antagonist on liver function impairment in immunized rabbits. Int J Med Med Sci 2010; 2: 395-401.

13. Tripathi T, Shahid M, Raza A et al. Dose-dependent effect of histamine on liver function markers in immunized rabbits. Exp Toxicol Pathol 2012; 64: 875-881.

14. Tripathi T, Shahid M, Khan HM et al. The Influence of Histamine H1-receptors on liver functions in immunized rabbits. Saudi J Biological Sci 2011; 18: 411-418.

15. Koganei A, Tsuchiya T, Samura $\mathbf{K}$ et al. Use of whole sheep red blood cells in ELISA to assess immunosuppression in vivo. J Immunotoxicol 2007; 4: 77-82.

16. Zhang H, Gou Y, Yuan J. Conjugated linoleic acid enhanced the immune function in broiler chicks. Br J Nutr 2005; 94: 746-752.

17. Bafna AR, Mishra SH. Actividad inmunomoduladora del extracto de methanol de las raíces de Cissampelos pareira Linn. Ars Pharm 2005; 46: $253-262$.

18. Lozeva V, Tuomisto L, Tarhanen J et al. Increased concentrations of histamine and its metabolite, tele-methylhistamine and down-regulation of histamine $\mathrm{H} 3$ receptor sites in autopsied brain tissue from cirrhotic patients who died in hepatic coma. J Hepatol 2003; 39: 522-527.
19. Arrang JM, Garbarg M, Schwartz JC et al. Auto-inhibition of brain histamine release mediated by a novel class (H3) of histamine receptor. Nature 1983; 302: 832-837.

20. Garbarg M, Tuong MD, Gros C et al. Effects of histamine H3receptor ligands on various biochemical indices of histaminergic neuron activity in rat brain. Eur J Pharmacol 1989; 164: 1-11.

21. Francis H, Franchitto A, Ueno $Y$ et al. H3 histamine receptor agonist inhibits biliary growth of BDL rats by downregulation of the cAMPdependent PKA/ERK1/2/ELK-1 pathway. Lab Invest 2007; 87: 473-487.

22. Schlicker E, Malinowska B, Kathmann M et al. Modulation of neurotransmitter release via histamine H3 heteroreceptors. Fundam Clin Pharmacol 1994; 8: 128-137.

23. Thapa BR, Walia A. Liver function tests and their interpretation. Indian J Pediatr 2007; 74: 663-671.

24. Fortson WC, Tedesco FJ, Starnes EC et al. Marked elevation of serum transaminase activity associated with extra hepatic biliary tract disease. J Clin Gastroenterol 1985; 7: 502-505.

25. Johnson RD, O'Connor ML, Kerr RM. Extreme serum elevations of aspartate aminotransferase. Am J Gastroenterol 1995; 90: 1244-1245.

26. Mathiesen UL, Franzén LE, Frydén A et al. The clinical significance of slightly to moderately increased liver transaminase values in asymptomatic patients. Scand J Gastroenterol 1993; 34: 85-91.

27. Hasan FAM, Owyed S. Interpretation of liver chemistry tests. Bull Kuwait Inst Med Spec 2003; 2: 27-31.

28. Yap CYF, Aw TC. Liver Function Tests (LFTs). Proceedings Singapore Healthcare 2010; 19: 80-82.

Received October 21, 2012 Accepted September 20, 2013. 